\title{
ON THE CHARACTERISTIC POLYNOMIAL OF THE PRODUCT OF SEVERAL MATRICES
}

\author{
WILLIAM E. ROTH
}

We shall prove two theorems.

TheOREM I. If $A$ is an $n \times n$ matrix with elements in the field $F$, if $R$ and $S_{i}, i=1,2, \cdots, r$, are $1 \times n$ matrices with elements in $F$, and $D_{i}=R^{T} S_{i}$, where $R^{T}$ is the transpose of $R$, and if the characteristic polynomial of $A_{i}=A+D_{i}$ is

$$
\left|x I-A_{i}\right|=m_{i 0}+m_{i 1} x+m_{i 2} x^{2}+\cdots+m_{i, r-1} x^{r-1},
$$

where $m_{i, j-1}, i, j=1,2, \cdots, r$, are polynomials in $x^{r}$ with coefficients in $F$, then the characteristic polynomial of the product $P=A_{1} A_{2} \cdots A_{r}$ is given by $(-1)^{(r-1) n}|\Delta(x)|$, where

$$
\Delta\left(x^{r}\right)=\left(\begin{array}{llll}
m_{10}, & m_{1, r-1} x^{r-1}, & m_{1, r-2} x^{r-2}, \cdots, m_{11} x \\
m_{21} x, & m_{20}, & m_{2, r-1} x^{r-1}, \ldots, m_{22} x^{2} \\
m_{32} x^{2}, & m_{31} x, & m_{30}, & \ldots, m_{33} x^{3} \\
\ldots & \ldots & \ldots & \ldots . . . . . . . . \\
m_{r, r-1} x^{r-1}, & m_{r, r-2} x^{r-2}, & m_{r, r-3} x^{r-3}, \ldots, m_{r, 0}
\end{array}\right) .
$$

This proposition has been proved by the writer [1] for the case $r=2$. Recently Parker [2] generalized that result and Goddard [3] gave an alternate proof of it and extended his method to the product of three matrices. This latter result does not come under the theorem above. Schneider [5] proved the theorem for the case $A_{i} A_{j}=0$, $i<j, i, j=1,2, \cdots, r$.

Capital letters and expressions in bold faced parentheses will indicate matrices with elements in the field $F$ or in $F(\omega)$, the extension of $F$ by the adjunction of $\omega$ a primitive $r$ th root of unity to it, and in $F(x)$ the polynomial domain of $F(\omega)$. The direct product of $B$ and $C$ is $\left(b_{i j} C\right)=B\langle C\rangle$. The product indicated by $\Pi$ will run from 1 to $r$.

If $R$ is not zero a nonsingular matrix $Q$ with elements in $F$ exists such that $Q R^{T}=(1,0, \cdots, 0)^{T}$; as a result

$$
Q D_{i} Q^{I}=(1,0, \cdots, 0)^{T} S_{i} Q^{I}=E_{i},
$$

where $Q^{I}$ is the inverse of $Q$ and $E_{i}$ has nonzero elements in only the first row. Now let

Presented to the Society, April 23, 1955; received by the editors April 4, 1955 and, in revised form, August 26, 1955. 


$$
Q A_{k} Q^{I}=M_{k}=Q\left(A+D_{k}\right) Q^{I}=M+E_{k},
$$

where $Q$ is the matrix defined above and $Q A Q^{I}=M=\left(m_{i j}\right)$. Consequently $M_{k}=\left(m_{i j}^{(k)}\right)$, where $m_{i j}^{(k)}=m_{i j}+e_{i j}^{(k)}$ and $m_{i j}^{(k)}=m_{i j}$ for $i>1$. That is, the matrices $M_{k}$ differ only in the elements of their first rows. As a result the elements of the first columns of the adjoints $\left[x I-M_{k}\right]^{A}$ and $[x I-M]^{A}$ of $x I-M_{k}$ and $x I-M$ respectively are identical for $k=1,2, \cdots, r$, since all these matrices agree in the elements of their last $n-1$ rows and for the same reason

$$
\begin{aligned}
& N_{k}(x)=\left[x I-M_{k}\right][x I-M]^{A},
\end{aligned}
$$

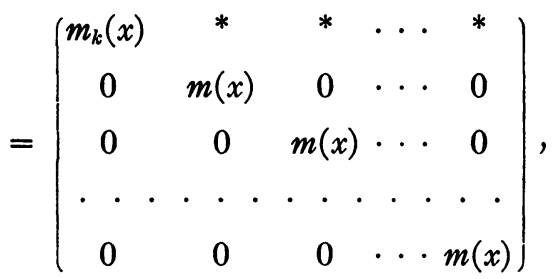

$k=1,2, \cdots, r$, where asterisks indicate nonzero elements in $F(x)$ and $|x I-M|=m(x)$.

Let

$$
W=\left(\omega_{i j}\right)=\left(\omega^{(i-1)(1-i)}\right), \quad i, j=1,2, \cdots, r
$$

then

$$
\left|W\left\langle I_{k}\right\rangle\right|=|W| k,
$$

where $I_{k}$ is the identity matrix of order $k$. The determinantal equation holds because $W\left\langle I_{k}\right\rangle$ can be transformed by the interchange of rows and corresponding columns to the direct sum $W \dot{+} W \dot{+} \cdots+\dot{+}$ of $k$ summands.

We shall operate in $F(x)$ on the matrix

$$
\begin{aligned}
& M(x)=\left(\delta_{i j} x I-\delta_{i+1, j} M_{i}\right) \quad\left(\delta_{r+1,1}=1\right)
\end{aligned}
$$

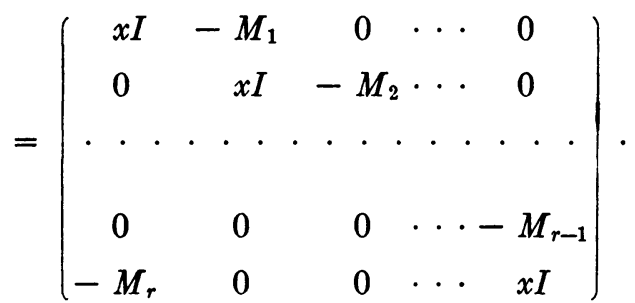

If we multiply this matrix on the right by one whose first row is $I$, $M_{1} x^{-1}, M_{1} M_{2} x^{-2}, \cdots, M_{1} M_{2} \cdots M_{r-1} x^{-r+1}$, whose second row is $0, I, M_{2} x^{-1}, \cdots, M_{2} M_{3} \cdots M_{r-1} x^{-r+2}$, and whose last row is 
$0,0,0, \cdots, I$, we find that the determinant of the product is $\left|x^{r} I-M_{r} M_{1} M_{2} \cdots M_{r-1}\right|$ and is therefore equal to $\left|x^{r}-P\right|$. The proof of Theorem I will consist in showing that

$$
|M(x)|=(-1)^{(r-1) n}\left|\Delta\left(x^{r}\right)\right| .
$$

We now proceed to establish this equation.

$$
\begin{aligned}
M(x) W\langle I\rangle & =\left(\delta_{i j} x I-\delta_{i+1, k} M_{i}\right)\left(\omega^{(k-1)(1-j)} I\right), \\
& =\left(\omega^{(i-1)(1-j)} x I-\omega^{i(1-j)} M_{i}\right), \\
& =\left(\omega^{1-j}\left\{\omega_{i j}\left[\omega^{j-1} x I-M_{i}\right]\right\}\right) .
\end{aligned}
$$

The number $\omega^{1-i}$ is a common multiplier of the $n \times n$ matrices in the $j$ th column of the $n r \times n r$ matrix in right member above. Consequently the determinant of this matrix has the factor $\pi \omega^{(1-k) n}=\omega^{-r(r-1) n / 2}$ $=\omega^{r(r-1) n / 2}=(-1)^{(r-1) n}$. The determinantal equation obtained from the matric equation above is as a result:

$$
|M(x)||W|^{n}=(-1)^{(r-1) n}\left|\omega_{i j}\left[\omega^{j-1} x I-M_{i}\right]\right| .
$$

According to (3) the product

$$
\left(\omega_{i k}\left[\omega^{k-1} x I-M_{i}\right]\right)\left(\delta_{k j}\left[\omega^{j-1} x I-M\right]^{A}\right)=\left(\omega_{i j} N_{i}\left(\omega^{j-1} x\right)\right) .
$$

The $n r \times n r$ matrix of the right member of this equation can be transformed by the interchange of corresponding rows and columns to a similar one having the form

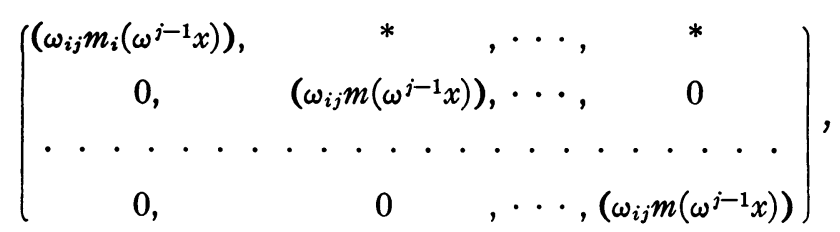

where asterisks represent $r \times r$ matrices with elements in $F(x)$ and the zeros are $r \times r$ zero matrices. The determinant of this matrix is

$$
|W|^{n-1}\left[\prod m\left(\omega^{j-1} x\right)\right]^{n-1}\left|\left(\omega_{i j} m_{i}\left(\omega^{j-1} x\right)\right)\right|
$$

for each of the matrices $\left(\omega_{i j} m\left(\omega^{j-1} x\right)\right)$ has $m\left(\omega^{j-1} x\right)$ as a divisor of all elements in the $j$ th column. The determinant of the direct sum $\left(\delta_{i j}\left[\omega^{i-1} x I-M\right]^{A}\right)$ in equation $(7)$ is $\left[\prod m\left(\omega^{j-1} x\right)\right]^{n-1}$; consequently the determinantal equation which follows from (7) and (8) is

$$
\begin{aligned}
& \left|\omega_{i j}\left[\omega^{j-1} x I-M_{i}\right]\right|\left[\prod m\left(\omega^{j-1} x\right)\right]^{n-1} \\
& \quad=|W|^{n-1}\left[\prod m\left(\omega^{j-1} x\right)\right]^{n-1}\left|\left(\omega_{i j} m_{i}\left(\omega^{j-1} x\right)\right)\right|,
\end{aligned}
$$

or

$$
\left|\omega_{i j}\left[\omega^{j-1} x I-M_{i}\right]\right|=|W|^{n-1}\left|\left(\omega_{i j} m_{i}\left(\omega^{j-1} x\right)\right)\right|
$$


where the determinant of the left member is that of an $n r \times n r$ matrix and those in the right members are of order $r$. From (6) and (9) we have $|M(x)|=(-1)^{(r-1) n}\left|\left(\omega_{i j} M_{i}\left(\omega^{j-1} x\right)\right)\right| /|W|$. It remains to be shown that the right member here is $(-1)^{(r-1) n}\left|\Delta\left(x^{r}\right)\right|$; this is easily accomplished by multiplying $\Delta\left(x^{r}\right)$ in (1) on the right by $W$. Herewith equation (5) is established and the proof of Theorem I is completed.

COROLlARY. Under the hypotheses of Theorem $\mathrm{I}$ and if $B$ is an $n \times n$ matrix with elements in $F$ and if $B_{i}=B+S_{i}^{T} R$ and

$$
\left|x I-B_{i}\right|=n_{i 0}+n_{i 1} x+n_{i 2} x^{2}+\cdots+n_{i, r-1} x^{r-1}
$$

then the characteristic polynomial of $B_{1} B_{2} \cdots B_{r}$ is given by $(-1)^{(r-1) n}\left|\Delta^{\prime}(x)\right|$ where

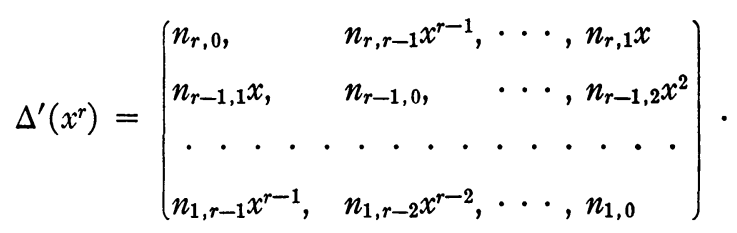

This case can be made to come under Theorem I for $B_{i}^{T}=B^{T}+R^{T} S_{i}$, where $B_{i}^{T}$ now satisfies the conditions imposed upon $A_{i}$. Moreover $\left|x I-B_{i}\right|=\left|x I-B_{i}^{T}\right|$. Since $\left(B_{1} B_{2} \cdots B_{r}\right)^{T}=B_{r}^{T} B_{r-1}^{T} \cdots B_{1}^{T}$, it follows that in $\Delta\left(x^{r}\right)$ of (1) we must replace the elements $m_{i, j-1} x^{j-1}$ by $n_{r-i+1, j-1} x^{j-1}$ in forming the matrix $\Delta^{\prime}\left(x^{r}\right)$ above. This proves the corollary.

Theorem II. If $D_{i}, i=1,2, \cdots, r$, are $n \times n$ matrices with elements in $F$, each of which is nilpotent and commutative with the others and with $A$, which also has elements in $F$, then the characteristic polynomials of $A_{i}=A+D_{i}, i=1,2, \cdots, r$, are given by

$$
|x I-A|=m_{0}+m_{1} x+m_{2} x^{2}+\cdots+m_{r-1} x^{r-1},
$$

where $m_{i-1}, i=1,2, \cdots, r$, are polynomials in $x^{r}$ with coefficients in $F$, and the characteristic polynomial of the product $P=A_{1} A_{2} \cdots A_{r}$ is $(-1)^{(r-1) n}|\bar{\Delta}(x)|$, where

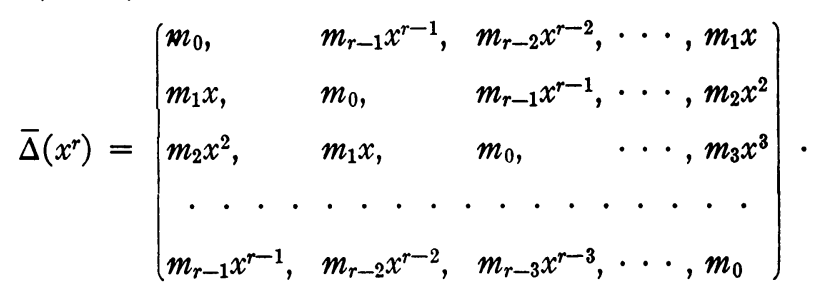

According to a theorem by Frobenius [4], the determinant of the 
matrix $B+C$ is equal to that of $B$ if $B$ and $C$ are commutative matrices and $C$ is nilpotent. This establishes equation (10) as giving the characteristic polynomial of $A_{i}, i=1,2, \cdots, r$. By Theorem I the determinant

$$
\left|x I-A^{r}\right|=(-1)^{(r-1) n}|\bar{\Delta}(x)| .
$$

We shall proceed by induction. Let $P_{i}=A_{1} A_{2} \cdots A_{i}$, then

$$
\left|x I-P_{1} A^{r-1}\right|=\left|x I-\left(A+D_{1}\right) A^{r-1}\right|=\left|x I-A^{r}-D_{1} A^{r-1}\right| .
$$

Now the matrix $D_{1} A^{r-1}$ is nilpotent and commutative with $x I-A^{r}$ consequently the determinants above are equal to $\left|x I-A^{r}\right|$. We assume that

$$
\left|x I-P_{i} A^{r-i}\right|=\left|x I-A^{r}\right|
$$

then

$$
\left|x I-P_{i+1} A^{r-i-1}\right|=\left|x I-P_{i} A^{r-i}-P_{i} D_{i+1} A^{r-i-1}\right| .
$$

Here $P_{i} D_{i+1} A^{r-i-1}$ is commutative with $x I-P_{i} A^{r-i}$ and is nilpotent because $D_{i+1}$ is nilpotent and commutative with both $P_{i}$ and $A$; hence by Frobenius' theorem

$$
\left|x I-P_{i+1} A^{r-i-1}\right|=\left|x I-P_{i} A^{r-i}\right|=\left|x I-A^{r}\right| .
$$

Consequently

$$
|x I-P|=\left|x I-A^{r}\right|=(-1)^{(r-1) n}|\bar{\Delta}(x)|,
$$

and the theorem is proved.

\section{REFERENCES}

1. W. E. Roth, On the characteristic polynomial of the product of two matrices, Proc. Amer. Math. Soc. vol. 5 (1954) pp. 1-3.

2. W. V. Parker, $A$ note on a theorem of Roth, Proc. Amer. Math. Soc. vol. 6 (1955) pp. 299-300.

3. L. S. Goddard, On the characteristic function of a matrix product, Proc. Amer. Math. Soc. vol. 6 (1955) pp. 296-298.

4. Frobenius, Preuss. Akad. Wiss. Sitzungsber. (1896) I pp. 601-614.

5. Schneider, $A$ pair of matrices having the property $\mathrm{P}$, Amer. Math. Monthly vol. 62 (1955) pp. 247-249.

Mississippi City, Miss. 